\title{
Preliminary Design of Haptic Icons from Users
}

\author{
Wonil Hwang and Dongsoo Kim \\ Department of Industrial and Information Systems Engineering, Soongsil University, Korea \\ \{wonil, dskim\} essu.ac.kr
}

\begin{abstract}
Haptic icons are useful for blind people as well as normal people to perceive information from their environments. Thus, lots of efforts were given to designing usable haptic icons, but not much progress was made in designing haptic icons so far, in terms of variety and intuitiveness. The purpose of this study is to investigate how to match vibrotactile stimuli with representational information or abstract concepts to design a variety of and intuitive haptic icons. We employed the bi-directional approach to ask users about their association between representational information/abstract concepts and perceived vibrotactile stimuli. Two-staged experiments were conducted with forty participants. From the experiments, verbal descriptions corresponding to each of 36 vibrotactile stimuli and drawings of vibration corresponding to each of 27 representational information/abstract concepts in the context of humancomputer interaction were collected. We can conclude that the associations that users described from these experiments would provide the foundation for designing more intuitive haptic icons in enough variety.
\end{abstract}

Keywords: Haptic icons, vibrotactile stimuli, representative information, abstract concepts, intuitiveness.

\section{Introduction}

Haptics comprises the studies related to delivering information based on senses of touch and to developing instruments to facilitate information delivery. Although visual and auditory channels have been considered as two main communication channels of human bodies, recently, the haptic channel based on vibrotactile stimuli has been actively studied to support or replace visual and auditory channels $[6,8]$. For example, haptic navigation aids for automotive drivers and surgical instruments based on haptic feedback are haptic applications as communication channels. And also, especially, the haptic devices as communication media help blind peoples to easily recognize information from their environments [3]. Thus, haptic channels are useful for both of normal people and blind people to perceive information from their environments.

Likewise visual and auditory displays, the haptic displays are utilized as a medium between users and information providers for effective and efficient communication. Visual and auditory displays may often employ visual and auditory icons for users to 
intuitively understand information [1]. In the same vein, haptic displays need haptic icons that are as much usable as visual and auditory icons. Even though lots of efforts were given to designing usable haptic icons [2, 4, 7], not much progress was made in designing haptic icons so far, in terms of variety and intuitiveness. Therefore, this study was motivated on how we design haptic icons that have intuitiveness and intimacy with users.

The purpose of this study is to investigate how to match vibrotactile stimuli with representational information or abstract concepts to design a variety of and intuitive haptic icons. Haptic icons should be distinguishable from each other and easily learned by users. So, this study tries to find what kinds of representational information or abstract concepts users associate with specific vibrotactile stimuli.

\section{Methods}

We employed the bi-directional approach to ask users about their association between representational information/abstract concepts and perceived vibrotactile stimuli: 1) asking users what kinds of representational information/abstract concepts are to be delivered by each vibrotactile stimulus; and 2) asking users to draw proper vibrotactile stimulus to deliver each representational information/abstract concepts. By this bi-directional approach, two-staged experiments were conducted and a total of forty participants took part in the experiments.

\subsection{Apparatus}

In order to generate vibrotactile stimuli that were used in the first stage, we utilized the vibration excitation system, which was designed to generate sinusoidal vibration with a range of frequency and amplitude. This vibration excitation system included the mini-shaker (Brüel and Kjær Type 4810) and a programmable function generator (Tabor Electronics WW5062) as the key components. The operating frequency range of the mini-shaker is DC - $18,000 \mathrm{~Hz}$, and the maximum bare table acceleration amounts to $550 \mathrm{~m} / \mathrm{s} 2(55.1 \mathrm{~g})$ in the frequency range of $65 \mathrm{~Hz}-4,000 \mathrm{~Hz}$. The programmable function generator was controlled by a waveform creation software (ArbConnection 4.x) in PC.

\subsection{Experiments}

In the first stage, 20 participants ( 8 males and 12 females; mean: 23.9 years old and standard deviation: 1.65 years old) took part in the experiment. They were presented a total of 36 distinguishable vibrotactile stimuli, which were generated by two kinds of combination: (1) Five types of frequency $(320,160,80,40,20 \mathrm{~Hz})$ and four types of rhythm (vibrating in $100 \%, 75 \%, 50 \%, 25 \%$ of a second) with $20 \mathrm{~dB}$ sinusoidal vibration; and (2) Five types of macro-wave pattern (horizontal, increasing, decreasing, increasing-decreasing, decreasing-increasing patterns) and four types of rhythm (vibrating in $100 \%, 75 \%, 50 \%, 25 \%$ of a second) with $160 \mathrm{~Hz}$ and $20 \mathrm{~dB}$ 
sinusoidal vibration. And considering human-computer interaction context they were asked to describe a proper representational information/abstract concept that was to be delivered by each vibrotactile stimulus after perceiving each of 36 distinguishable vibrotactile stimuli in random order.

In the second stage, 27 representational information/abstract concepts were selected by the results of the first stage experiments and other 20 participants (10 males and 10 females; mean: 23.9 years old and standard deviation: 1.62 years old) participated in the experiments. They were asked to freely draw a proper vibrotactile stimulus with frequency, rhythm and macro-wave pattern that was to deliver each of 27 representational information/abstract concepts in random order.

\section{Results}

The data resulted from the two-staged experiments were quite qualitative, because we collected the verbal descriptions corresponding to each of 36 vibrotactile stimuli from the first stage experiments and drawings of vibration corresponding to each of 27 representational information/abstract concepts in the context of human-computer interaction from the second stage experiments. Despite such qualitative data we tried to analyze them in a way similar to quantitative analysis by counting the frequency of verbal descriptions and coding the drawings with 3-digit numbers.

\subsection{First Stage Experiments}

From the first stage experiments, 20 participants represented each of 36 vibrotactile (or haptic) stimuli as 15 to 28 descriptions that were used in the context of humancomputer interaction. Because many of these descriptions were duplicated one another in their meaning, we grouped them and selected most frequent 27 descriptions as representative information/abstract concepts for vibrotactile (or haptic) stimuli (see the first column of Table1).

\subsection{Second Stage Experiments}

From the second stage experiments, 20 participants drew a vibration for each of 27 representative information/abstract concepts, and we coded them with 3-digit numbers (see Figure 1 and 2) as follows: (1) First digit: frequency type (1: high, 2: middle, 3: low); (2) second digit: macro wave type (1: horizontal, 2: increasing, 3: decreasing, 4: increasing \& decreasing, 5: decreasing \& increasing, 6: stepping down, 7: stepping up); and (3) third digit: rhythm type (1: vibrating in $100 \%, 2$ : vibrating in $75 \%$, 3: vibration in $50 \%$, 4: vibrating in $25 \%$ of a second). And then we made a frequency table per representative information/abstract concept, and resulted in 
recommended haptic stimuli for each of representative information/abstract concept based on the highest frequency (see the second column in Table 1). We calculated Herfindahl-Hirschman Index (HHI), which indicated the concentration ratio (originally, it measures the amount of competition in the market) [5], and used it as a measure of consensus among 20 participants about haptic stimuli for each of representative information/abstract concept (see the last two columns in Table 1).

As shown in Table 1, 15 distinguishable haptic stimuli are recommended to represent specific information/abstract concepts, even though some of recommended haptic stimuli repeatedly appear for representative information/abstract concepts. For example, '211', which is coded for vibration with middle frequency, horizontal macro wave type and vibrating in $100 \%$ of a second, appears for 8 representative information/abstract concepts. If we apply 'normalized HHI $>0.025$ ' to find relatively good consensus among 20 participants about haptic stimuli for each of representative information/abstract concept, we can find that there are good consensus for 12 representative information/abstract concepts, such as clicks/selecting, dragging/moving, detecting virus, program stopped/no response, error, completing actions, inputting data/typing, double-clicks/executing, in the middle of action/buffering/installing, deleting, alarm/message, booting.

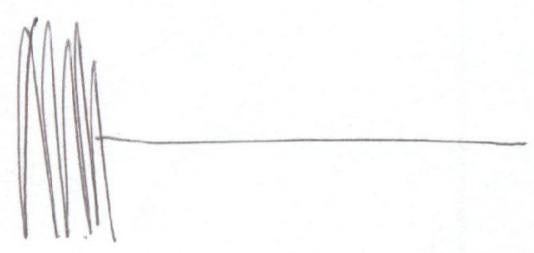

Fig. 1. An example of drawing coded as '214' and representing 'clicks/selecting'

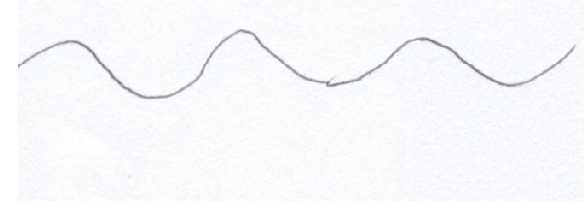

Fig. 2. An example of drawing coded as ' 311 ' and representing 'dragging/moving' 
Table 1. Drawing results of haptic stimuli

\begin{tabular}{|c|c|c|c|c|}
\hline $\begin{array}{l}\text { Information delivered } \\
\text { by haptic stimuli }\end{array}$ & $\begin{array}{l}\text { Recommended } \\
\text { haptic stimuli }\end{array}$ & $\begin{array}{l}\text { Percent of votes } \\
\text { for a recommended } \\
\text { haptic stimulus }\end{array}$ & HHI & $\begin{array}{c}\text { Normalized } \\
\text { HHI }\end{array}$ \\
\hline Clicks/Selecting & 214 & $45.0 \%$ & 0.2600 & 0.1675 \\
\hline Dragging/Moving & 311 & $28.6 \%$ & 0.1338 & 0.0616 \\
\hline Detecting virus & 211 & $22.7 \%$ & 0.1446 & 0.0496 \\
\hline $\begin{array}{l}\text { Program stopped/No } \\
\text { response }\end{array}$ & 214 & $25.0 \%$ & 0.1150 & 0.0469 \\
\hline Error & 211,213 & $20.0 \%$ & 0.1150 & 0.0413 \\
\hline Completing actions & 211 & $22.7 \%$ & 0.0992 & 0.0391 \\
\hline Inputting data/Typing & 213 & $20.0 \%$ & 0.1150 & 0.0345 \\
\hline $\begin{array}{c}\text { Double- } \\
\text { clicks/Executing }\end{array}$ & 213 & $20.0 \%$ & 0.1150 & 0.0345 \\
\hline $\begin{array}{c}\text { In the middle of } \\
\text { action/Buffering/Installing }\end{array}$ & 211,311 & $18.2 \%$ & 0.1033 & 0.0343 \\
\hline Deleting & 133 & $20.0 \%$ & 0.1200 & 0.0320 \\
\hline Alarm/Message & 213 & $20.0 \%$ & 0.1050 & 0.0304 \\
\hline Booting & 213 & $20.0 \%$ & 0.0950 & 0.0254 \\
\hline $\begin{array}{l}\text { New windows/Pop- } \\
\text { ups }\end{array}$ & 214 & $20.0 \%$ & 0.0900 & 0.0250 \\
\hline $\begin{array}{c}\text { Sending-receiving } \\
\text { files/Up-loading/Down- } \\
\text { loading }\end{array}$ & 211 & $20.0 \%$ & 0.0850 & 0.0240 \\
\hline $\begin{array}{l}\text { Closing programs or } \\
\text { windows }\end{array}$ & $133,211,214$ & $13.6 \%$ & 0.0868 & 0.0215 \\
\hline Locking up & 114 & $18.2 \%$ & 0.0785 & 0.0209 \\
\hline Scrolling down & 214,311 & $15.0 \%$ & 0.0900 & 0.0200 \\
\hline Undoing & 114,134 & $15.0 \%$ & 0.0850 & 0.0196 \\
\hline Warning & 111,212 & $15.0 \%$ & 0.0950 & 0.0196 \\
\hline Computer shut-down & 211 & $15.0 \%$ & 0.0800 & 0.0143 \\
\hline $\begin{array}{l}\text { Creating folders, files } \\
\text { or shortcuts }\end{array}$ & 221 & $15.0 \%$ & 0.0800 & 0.0143 \\
\hline
\end{tabular}

1 Three-digit means 'frequency type - macro wave type - rhythm type': (1) Frequency type (1: high, 2: middle, 3: low); (2) macro wave type (1: horizontal, 2: increasing, 3: decreasing, 4: increasing \& decreasing, 5: decreasing \& increasing, 6: stepping down, 7: stepping up); (3) rhythm type (1: vibrating in $100 \%$, 2: vibrating in $75 \%$, 3 : vibration in $50 \%$, 4 : vibrating in $25 \%$ of a second). 
Table 1. (Continued)

\begin{tabular}{ccccc}
\hline $\begin{array}{c}\text { Information delivered } \\
\text { by haptic stimuli }\end{array}$ & $\begin{array}{c}\text { Recommended } \\
\text { haptic stimuli }\end{array}$ & $\begin{array}{c}\text { Percent of votes } \\
\text { for a recommended } \\
\text { haptic stimulus }\end{array}$ & HHI & $\begin{array}{c}\text { Normalized } \\
\text { HHI }\end{array}$ \\
\hline Switching windows & 214 & $15.0 \%$ & 0.0800 & 0.0143 \\
Minimizing windows & 331 & $15.0 \%$ & 0.0750 & 0.0133 \\
Printing & Six stimuli & $10.0 \%$ & 0.0800 & 0.0092 \\
$\begin{array}{c}\text { Refreshing } \\
\text { Connecting internet or } \\
\text { messenger }\end{array}$ & Four stimuli & $9.1 \%$ & 0.0620 & 0.0068 \\
Maximizing windows & $211,212,222$ & $10.0 \%$ & 0.0650 & 0.0066 \\
\hline
\end{tabular}

\section{Conclusions and Discussions}

From the two-staged experiments, we found what kinds of association between representational information/abstract concepts and vibrotactile stimuli users had in mind. First, from the first stage experiments we could select a total of 27 representative information/abstract concepts related to human-computer interaction from 36 distinguishable vibrotactile (or haptic) stimuli. It means that these 27 concepts are good candidates for information that can be delivered by haptic stimuli. Second, from the second stage experiments we could find which haptic stimuli could show relatively good representation for each of 27 concepts. Third, by introducing Herfindahl-Hirschman Index as a measure of consensus we could show how much users agreed that each of representative information /abstract concepts could be delivered by specific haptic stimuli. For example, 'clicks/selecting' is the representative information/abstract concept that users give the biggest consensus. Even though this study has some limitations as a preliminary study, including small sample size, the associations that users described from these experiments would provide the foundation for designing more intuitive haptic icons in enough variety.

Acknowledgements. This research was supported by Basic Science Research Program through the National Research Foundation of Korea(NRF) funded by the Ministry of Education, Science and Technology(2011-0013713).

\section{References}

1. Blattner, M.M., Sumikawa, D.A., Greenberg, R.M.: Earcons and icons: Their structure and common design principles. Human-Computer Interaction 4, 11-44 (1989) 
2. Brewster, S.A., Brown, L.M.: Tactons: Structured tactile messages for non-visual information display. In: Proc. of the 5th Australasian User Interface Conference, Sydney, Australia, pp. 15-23 (2004)

3. Colwell, C., Petrie, H., Kornbrot, D., Hardwick, A., Furner, S.: Haptic virtual reality for blind computer users. In: Proc. of the 3rd International ACM Conference on Assistive Technologies, New York, NY, USA, pp. 92-99 (1998)

4. Enriquez, M., MacLean, K.E., Chita, C.: Haptic phonemes: Basic building blocks of haptic communication. In: Proc. of the 8th International Conference on Multimodal Interfaces, Los Alamitos, CA, USA, pp. 302-309 (2006)

5. Hirschman, A.O.: The paternity of an index. The American Economic Review 54(5), 761-762 (1964)

6. Hwang, J., Hwang, W.: Vibration perception and excitatory direction for haptic devices. Journal of Intelligent Manufacturing 22, 17-27 (2011)

7. Hwang, J., Hwang, W.: Generation of effective vibrotactile stimuli to convey information. ICIC Express Letters 7(5), 1637-1641 (2013)

8. Ji, Y.G., Lee, K., Hwang, W.: Haptic perceptions in the vehicle seat. Human Factors and Ergonomics in Manufacturing \& Service Industries 21(3), 305-325 (2011) 